\title{
COMPACTIFICATION OF MAPPINGS
}

\author{
GEORGE L. CAIN, JR.
}

1. Introduction. A mapping (continuous function) $f: X \rightarrow f(X)=Y$ is compact if it is closed and point inverses are compact. (These mappings are of ten called perfect, or proper.) A compact mapping has the property that the inverse image of any compact set is compact. This property is sometimes taken as the definition of a compact mapping, although the two definitions are not equivalent for arbitrary range $Y$. Exact conditions for their equivalence are given in reference [6].

A compactification of $f$ is a pair $\left(X^{*}, f^{*}\right)$ where $X^{*}$ is a Hausdorff space containing $X$ as a dense subspace, and $f^{*}$ is a compact mapping of $X^{*}$ onto $Y$ such that $f^{*} \mid X=f$. G. T. Whyburn introduced the notion of a mapping compactification in 1953 when he showed that every mapping of one locally compact Hausdorff space onto another is a partial mapping of a compact mapping on a Hausdorff space [4], [7]. He also noted in reference [7] that a compactification for any mapping from one completely regular space onto another can be obtained by restricting the extension $\bar{f}: \beta X \rightarrow \beta Y$ to $\bar{f}^{-1}(Y)$.

In this paper we study some general properties of mapping compactifications and construct a class of so-called filter space compactifications of a mapping from a completely regular space onto a regular space. Each of these compactifications is associated in a natural way with a compactification of the domain of $f$. For $Y$ locally compact, we show that the domain $X$ is completely regular if and only if $f$ has a compactification; and if $X$ is completely regular, every compactification of $f$ is a filter space compactification. We shall assume that all spaces are Hausdorff. Thus in this paper, regular spaces, completely regular spaces, etc., are Hausdorff.

2. Mapping compactifications. Let $R$ denote the collection of all points $p$ in $Y$ having the property that there is a filter $\mathbf{F}$ on $X$ with no accumulation point such that $f(\mathbf{F})$ converges to $p$. The following result then follows directly from Theorem 1, p. 101, of [1].

Proposition 2.1. The restriction of $f$ to $f^{-1}(Y-R)$ is compact, and if $f$ is compact, $R$ is empty.

Presented to the Society, January 23, 1969; received by the editors January 3 , 1969. 
In case $X$ is regular, we need only consider open filters; that is, those which have a base consisting of open sets. Specifically, we have

Proposition 2.2. If $X$ is regular, $R$ consists of those points $p$ in $Y$ having the property that there is an open filter $G$ on $X$ with no accumulation point which is such that $f(G)$ converges to $p$.

Proof. Suppose $p$ is in $R, F$ has no accumulation point, and $f(F)$ converges to $p$. Let $v$ be the collection of all closed neighborhoods in $X$ which have a member of $F$ contained in their complement. The fact that $F$ has no accumulation point guarantees that each $x$ in $X$ has at least one neighborhood in $v$.

The collection $Q=\left\{X-V_{\alpha} \mid V_{\alpha} \in \mathcal{V}\right\}$ is a filter base. If $\boldsymbol{A}$ denotes the filter generated by $a$, then clearly $G=\left\{A \cap f^{-1}(U) \mid A \in A, U\right.$ is a neighborhood of $p\}$ is an open filter having no accumulation point, and $f(\boldsymbol{G})$ converges to $p$.

We shall let $S$ denote the singular set of $f$. ( $S$ is the collection of all $p$ in $Y$ such that in every neighborhood of $p$ there is a compact set with a noncompact inverse image [2], [5].) It follows easily from the fact that $f$ restricted to $f^{-1}(Y-\operatorname{cl}(R))$ is compact and the results in reference [6] that $S \subset \mathrm{cl}(R)$, and that if $Y$ is a $k$-space then $R \subset S=\operatorname{cl}(R)$.

Theorem 2.3. If $\left(X^{*}, f^{*}\right)$ is a compactification of $f$, then $f^{*}\left(X^{*}-X\right)=R$.

Proof. Let $p$ be in $R$ and suppose $F$ is a filter on $X$ such that $f(F)$ converges to $p$ and $F$ has no accumulation point in $X . f^{*}$ is compact, so there is an accumulation point $x$ of $F$ in $X^{*}-X$, and from the continuity of $f$ it follows that $f^{*}(x)=p$.

For $x$ in $X^{*}-X$, let $\boldsymbol{N}$ be the neighborhood filter of $x$. Since $X$ is dense in $X^{*}, F=\{N \cap X \mid N \in N\}$ is a filter on $X$ without an accumulation point in $X$ and is such that $f(F)$ converges to $f^{*}(x)$.

Corollary. If $X$ is locally compact, $R$ is closed. If in addition, $Y$ is a $k$-space, then $R=S$.

Proof. If $X$ is locally compact, $X$ is open in $X^{*}$. Thus $f^{*}\left(X^{*}-X\right)$ is closed.

A relation $\geqq$ is defined on the collection of all compactifications of $f$ by agreeing that $\left(X_{2}, f_{2}\right) \geqq\left(X_{1}, f_{1}\right)$ if there is a mapping $h$ of $X_{2}$ onto $X_{1}$ which leaves the points of $X$ fixed and is such that $f_{2}=f_{1} h$. If the mapping $h$ can be taken to be a homeomorphism, $\left(X_{1}, f_{1}\right)$ and $\left(X_{2}, f_{2}\right)$ are said to be equivalent. It is easy to verify that the relation $\geqq$ is a partial order and that $\left(X_{1}, f_{1}\right)$ and $\left(X_{2}, f_{2}\right)$ are equivalent if and only if the relations $\left(X_{2}, f_{2}\right) \geqq\left(X_{1}, f_{1}\right) \geqq\left(X_{2}, f_{2}\right)$ hold. 
In case $X$ and $Y$ are locally compact, Whyburn's unified space of the mapping $f$ provides a compactification $\left(X_{w}, f_{w}\right)$ in which $f_{w} \mid\left(X_{w}-X\right)$ is a homeomorphism onto $R[4]$. The next result shows that any compactification with this property precedes all others with respect to the partial order $\geqq$. Thus all such compactifications of $f$ are equivalent.

Theorem 2.4. Suppose the domain of $f$ is locally compact. If $\left(X_{w}, f_{w}\right)$ is a compactification of $f$ such that $f_{w}$ restricted to $X_{w}-X$ is a homeomorphism, then $\left(X^{*}, f^{*}\right) \geqq\left(X_{w}, f_{w}\right)$ for any compactification $\left(X^{*}, f^{*}\right)$ of $f$.

Proof. Define the function $h: X^{*} \rightarrow X_{w}$ by setting $h(x)=x$ for $x$ in $X$ and $h(x)=\left[f_{w}^{-1} f^{*}(x)\right] \cap\left(X_{w}-X\right)$ for $x$ in $X^{*}-X$. It is clear that $h$ is a function, that $f^{*}=f_{w} h$, and that $h$ is continuous at all points of $X$. To show that $h$ is continuous at a point $x$ in $X^{*}-X$, let $N$ be the neighborhood filter of $x$. Then $f^{*}(\boldsymbol{N})$ converges to $f^{*}(x)$, and $f_{w}$ is compact, so $h(\boldsymbol{N})$ has at least one accumulation point $z$. From the fact that $X$ is open in $X_{w}$ and $X^{*}$, and $h$ is the identity on $X$, it follows that $z$ can not be in $X$, so $h(N)$ has exactly one accumulation point $z=h(x)$ in $X_{w}-X$.

Let $W$ be a neighborhood of $z$. If every member of $h(\boldsymbol{N})$ meets the complement of $W$, then $G=\left\{G \cap\left(X_{w}-W\right) \mid G \in h(N)\right\}$ is a filter on $X_{w}$ with no accumulation point, and $f_{w}(G)$ converges to $y$. This contradicts the fact that $f_{w}$ is compact. Thus $h(\boldsymbol{N})$ converges to $z=h(x)$ and $h$ is continuous.

3. Filter space compactifications. Consider a mapping $f: X \rightarrow f(X)$ $=Y$, where $X$ is completely regular and $Y$ is regular. Let $\tilde{X}$ be a compactification (Hausdorff) of the space $X$. For $y \in Y$, let $\tilde{C}(y) \subset \tilde{X}$ be the collection of all accumulation points in $\tilde{X}$ of the inverse image of the neighborhood filter of $y$. Note that $f^{-1}(y) \subset \widetilde{C}(y)$ and $f^{-1}(y)$ $=\tilde{C}(y)$ if and only if $y \in Y-R$.

With the above assumptions and terminology, we now have the following theorem.

THEOREM 3.1. The mapping $f$ has a compactification $\left(X^{*}, f^{*}\right)$ with the property that for each $y \in Y$, there is a homeomorphism $h_{\nu}$ of $f^{*-1}(y)$ onto $\tilde{C}(y)$ which leaves the points of $f^{-1}(y)$ fixed.

We shall construct $\left(X^{*}, f^{*}\right)$ and establish via a series of propositions that it is a compactification of $f$ having the property in the theorem.

In constructing $\left(X^{*}, f^{*}\right)$, extensive use of the idea of a filter space as developed by F. J. Wagner [3] will be made. If $\tilde{X}$ is a collection of open filters on $X$ which includes the neighborhood filter of every $x$ 
in $X$, then $\tilde{X}$ can be made into a topological space by taking the collection of sets $\tilde{U}=\{F \in \tilde{X} \mid U \in F, U$ is an open subset of $X\}$ as a base for open sets in $\tilde{X}$. The space $\tilde{X}$ is known as a filter space over $X$. It is shown in reference [3] that the function $e: X \rightarrow e(X) \subset \tilde{X}$ defined by $e(x)=$ the neighborhood filter of $x$, is a homeomorphism and $e(X)$ is a dense subspace of $\tilde{X}$.

If $\tilde{X}$ is a compactification of the space $X$, we may consider $\tilde{X}$ to be a filter space over $X$ by associating with each point in $\tilde{X}$ the trace on $X$ of its neighborhood filter (Theorem 2 of [3]). For each filter $F$ in $\tilde{X}$, consider $Q_{y}=\left\{F \cap f^{-1}\left(W_{y}\right) \mid F \in F, W_{y}\right.$ a neighborhood of $\left.y\right\}$. Each $Q_{y}$ that does not contain the empty set is a base for a filter on $X$, which we shall denote by $F_{y}$. Note that $F_{y}$ is defined if and only if $F \cap f^{-1}\left(W_{y}\right) \neq \varnothing$ for every neighborhood $W_{y}$ of $y$ and every $F \in F$.

To avoid confusion, we shall throughout use the terminology " $F$ is a filter on $X$ " when $F$ is a collection of subsets of $X$, and " $F$ is a filter in $X$ " when $F$ is a member of $X$.

Proposition 3.2. For $F$ and $G$ in $\tilde{X}, F_{y}=G_{z}$ if and only if $F=G$, and $y=z$.

Proof. The "if" part is trivial. Assume $F_{y}=G_{z}$. The inverse image of every neighborhood of $y$ is a member of $F_{y}$ and the inverse image of every neighborhood of $z$ is a member of $G_{z}$, so it follows from the fact that $Y$ is Hausdorff that $y=z$. That $F=G$ follows from the fact that $F \subset F_{y}, G \subset G_{z}$, and the Hausdorff property of $\tilde{X}$.

It is clear that each $F_{y}$ is an open filter and that for $F=N_{x}$, the neighborhood filter of $x$, then $F_{y}=N_{x}$, where $y=f(x)$. Let $X^{*}$ denote the filter space consisting of all such $F_{y}$. Define the function $f^{*}$ from $X^{*}$ onto $Y$ by setting $f^{*}\left(F_{y}\right)=y$. Then $f^{*} \mid X=f$ and $X$ is dense in $X^{*}$.

Proposition 3.3. $f^{*}$ is continuous.

Proof. Let $R$ be a neighborhood of $y=f^{*}\left(F_{y}\right)$ and choose $W$ to be an open neighborhood of $y$ such that $\mathrm{cl}(W) \subset R$. Now $U=f^{-1}(W)$ is a member of $F_{y}$, so $U^{*}=\left\{G_{z} \in X^{*} \mid U \in G_{z}\right\}$ is a neighborhood of $F_{y}$. For any $\boldsymbol{G}_{z} \in U^{*}, f^{-1}(W) \in G_{z}$, so $z=f^{*}\left(\boldsymbol{G}_{z}\right) \in \operatorname{cl}(W) \subset R$.

\section{Proposition 3.4. $X^{*}$ is regular.}

Proof. Let $F_{y}$ and $G_{z}$ be two distinct points in $X^{*}$. If $y \neq z$, the existence of disjoint neighborhoods of the two points follows from the continuity of $f^{*}$ and the Hausdorff property of $Y$. If $F=G$, there are disjoint open sets $U$ and $V$ in $X$ such that $U \in F$ and $V \in G$ since $\tilde{X}$ is Hausdorff. Thus $U^{*}$ and $V^{*}$ are disjoint neighborhoods of $F_{y}$ and $G_{z}$, respectively. 
Next, let $F_{y} \in U^{*}$, and choose an open $F \in F$ and a neighborhood $W_{y}$ of $y$ so that $F \cap f^{-1}\left(W_{y}\right) \subset U$. Let $M$ be an open neighborhood of $y$ such that $\operatorname{cl}(M) \subset W_{y}$. The space $\tilde{X}$ is regular, so choose an open $V \subset X$ so that $V \in F$ and $\operatorname{cl}(\tilde{V}) \subset \tilde{F}$, where $\tilde{V}$ denotes the collection of all elements in $\tilde{X}$ that have $V$ as a member. Let $R=V \cap f^{-1}(M)$. We shall show that $R^{*}$ is a neighborhood of $F_{y}$ such that $\operatorname{cl}\left(R^{*}\right) \subset U^{*}$.

First note that since $V \in F$, we have $V \cap f^{-1}(M)=R \in F_{y}$, so $R^{*}$ is indeed a neighborhood of $F_{y}$. If $G_{z} \in \operatorname{cl}\left(R^{*}\right)$, every open set in $G_{z}$ meets $R$, so $z \in \operatorname{cl}(M) \subset W_{y}$. Let $W_{z}$ be a neighborhood of $z$ such that $W_{z} \subset W_{y}$. For every $G \in G$, we have $\left[G \cap f^{-1}\left(W_{z}\right)\right] \cap R \neq \varnothing$, and hence $G \cap V \neq \varnothing$ for every $G$ in $G$. This means that $G \in \operatorname{cl}(\tilde{V}) \subset \tilde{F}$, or $F \in G$. Hence $F \cap f^{-1}\left(W_{z}\right) \in G_{z}$, and $U \in G_{z}$ since $F \cap f^{-1}\left(W_{z}\right) \subset U$.

Proposition 3.5. If $\mathcal{F}$ is an open filter on $X^{*}$ such that $f^{*}(\mathfrak{F})$ converges to some $y$ in $Y$, then $F$ has an accumulation point.

Proof. $F \cap X=\{A \cap X \mid A \in \mathfrak{F}\}$ is an open filter base on $X$ since $X$ is dense in $X^{*}$. If we consider $\mathfrak{F} \cap X$ to be a filter base on $\tilde{X}$, it has an accumulation point $F \in \tilde{X}$. Suppose $F \cap f^{-1}\left(W_{y}\right)=\varnothing$ for some $F$ in $F$ and neighborhood $W_{y}$ of $y$. Choose $A$ in $\mathcal{F}$ such that $f^{*}(A) \subset W_{y}$. Now $F \cap(A \cap X) \neq \varnothing$ by Lemma 3 of reference [3]; but for $x \in F$ $\cap(A \cap X)$, the neighborhood filter $\boldsymbol{N}_{x}$ is a member of $A$ and $f^{*}\left(\boldsymbol{N}_{x}\right)$ $=f(x) \in W_{y}$, so $f^{-1}\left(W_{y}\right) \cap F \neq \varnothing$, a contradiction. Thus $F \cap f^{-1}\left(W_{y}\right) \neq \varnothing$ for every $F$ in $F$ and every neighborhood $W_{y}$ of $y$.

We show that $F_{y}$ is an accumulation point of $\mathcal{F}$. If not, there is an open $U \subset X$ such that $U \in F_{y}$ and $U^{*} \cap A_{1}=\varnothing$ for some $A_{1}$ in $\mathcal{F}$. Choose $F$ in $F$ and a neighborhood $W_{y}$ of $y$ so that $F \cap f^{-1}\left(W_{y}\right) \subset U$. Let $A_{2}$ in $\mathcal{F}$ be such that $f^{*}\left(A_{2}\right) \subset W_{y}$, and define $A=A_{1} \cap A_{2}$. Then for any $x \in A \cap X$, it is true that $f(x) \in W_{y}$. Or in other words, $A \cap X$ $\subset f^{-1}\left(W_{y}\right)$, which contradicts our statement that $U^{*} \cap A_{1}$ is empty.

It is clear from (2.1) and (2.2) that $f^{*}$ is a compact mapping, so $\left(X^{*}, f^{*}\right)$ is a compactification of $f$. The next proposition completes the proof of Theorem 3.1.

Proposition 3.6. For each $y \in Y$, there is a homeomorphism $h_{y}$ of $f^{*-1}(y)$ onto $\tilde{C}(y)$ which leaves the points of $f^{-1}(y)$ fixed.

Proof. Let $h: X^{*} \rightarrow \tilde{X}$ be defined by $h\left(F_{y}\right)=F$. To show that $h$ is continuous, let $U$ be an open set in $X$ such that $U \in F=h\left(F_{y}\right)$. Choose an open set $V \in F$ so that $\operatorname{cl}(\tilde{V}) \subset \tilde{U}$. We know that $F \subset F_{y}$, so $V^{*}$ is a neighborhood of $F_{y}$. Suppose $G_{z} \in V^{*}$; then $V \in G_{z}$, and $V \cap G \neq \varnothing$ for every $G \in G$, which means that $G \in \operatorname{cl}(\tilde{V}) \subset \widetilde{U}$. Thus $h$ is continuous.

For each $y \in Y$, let $h_{y}=h \mid f^{*-1}(y)$. It follows from (3.2) that $h_{y}$ is one to one, and thus a homeomorphism since $f^{*-1}(y)$ is compact. That the 
range of $h_{y}$ is $\tilde{C}(y)$ is a consequence of the fact that $F_{y}$ is defined if and only if every member of $F$ meets the inverse image of every neighborhood of $y$.

Finally, we show that any compactification $\left(X^{\prime}, f^{\prime}\right)$ of $f$ with $X^{\prime}$ completely regular is a filter space compactification corresponding to some compactification $\tilde{X}$ of the space $X$. We have

Theorem 3.7. Suppose $\left(X^{\prime}, f^{\prime}\right)$ is a compactification of $f$ with $X^{\prime}$ completely regular, and let $\tilde{X}$ be a compactification of $X^{\prime}$, and hence of $X$. Then the corresponding filter space compactification $\left(X^{*}, f^{*}\right)$ is equivalent to $\left(X^{\prime}, f^{\prime}\right)$.

Proof. We have $X \subset X^{\prime} \subset \tilde{X}$, and will consider $\tilde{X}$ (and hence $X^{\prime}$ ) to be a filter space over $X$. Note that the filter $F$ on $X^{\prime}$ converges to the point $F \in X^{\prime}$, so that for any $F \in X^{\prime}$, we have $F_{y} \in X^{*}$, where $y=f^{\prime}(F)$.

Let $F_{y}$ be an element of $X^{*}$. If we consider $F_{y}$ to be a filter on $X^{\prime}$, it has an accumulation point $G \in X^{\prime}$, since $f\left(F_{y}\right)=f^{\prime}\left(F_{y}\right)$ converges to $y$. Any open set in $X$ which is a member of $G$ must meet every member of $F$, so it follows that $F=G$ since $\tilde{X}$ is Hausdorff. Thus $f^{\prime}(F)=y$. It now follows that $f(F)$ converges to $y$, so that $F_{y}=F$. Thus $X^{*}=X^{\prime}$ and $f^{*}=f^{\prime}$.

\section{REFERENCES}

1. N. Bourbaki, General topology. Part 1, Addison-Wesley, Reading, Mass., 1966.

2. G. L. Cain, Jr., Compact and related mappings, Duke Math. J. 33 (1966), 639645 .

3. F. J. Wagner, Notes on compactification. I, Nederl. Akad. Wetensch. Proc. Ser. A. 60(1957), 171-176.

4. G. T. Whyburn, A unified space for mappings, Trans. Amer. Math. Soc. 74(1953), 344-350.

5. - On compactness of mappings, Proc. Nat. Acad. Sci. U.S.A. 52(1964), $1426-1431$.

6. - Directed families and closedness of functions, Proc. Nat. Acad. Sci. U.S.A. 54 (1965), 688-692.

7. - Compactification of mappings, Math. Ann. 166(1966), 168-174.

Georgia Institute of Technology 